\title{
An Enhanced Fast Handover Using Hierarchical Setup for Mobile IP
}

\author{
V.Berlin Hency ${ }^{1,}$ Christina J. ${ }^{2}$, Dhushanthini A. ${ }^{2}$, Aiswariya V.T. ${ }^{2}$, Dr.D.Sridharan ${ }^{3}$ First A.
}

\begin{abstract}
Mobile IP protocol has been designed to handle global and seamless user's mobility on the Internet. However, Mobile IP suffers from handoff latency due to slow handoff discovery and binding association notification. To reduce this latency, we propose a new handoff protocol where we attribute a high priority in using hierarchical setup with fast handoff scheme. We study the influence of various system parameters on the performance of the two handoff protocols. We present an analytical model for handoff latency for both existing and our new scheme and conduct several simulations to compare and contrast the two handoff protocols. Our analytical study and simulation results provide a significant decrease of handoff latency and there by improvises the smoothness of handoffs.
\end{abstract}

Index Terms-Mobility, mobile IPv6, handoff latency, packet delay, fast handover scheme, hierarchical setup.

\section{INTRODUCTION}

With the development of Internet technology, the number of Internet users has been drastically increasing and users expect to connect to the Internet from "anywhere" at "anytime". The mobile devices moving with the user together constitute a personal area network which is a small scale mobile network. In recent years protocols such as Mobile IPv4 (MIP) and Mobile IPv6 (MIPv6) for supporting seamless connectivity to mobile hosts are developed. To support network mobility extending the existing protocols or developing new ones to support network mobility in an IPv6 network is being done.

One of the most important challenges in Mobile IPv6 is to provide the service for a mobile node to maintain its connectivity to the Internet when it moves from one domain to another, which is referred to as handover[1]. Here we deal with the fast handover problem, which is to provide rapid handover service for the delay-sensitive and real-time applications. Mobile IPv6 (MIPv6) is proposed to enable network applications to continuously operate at the required quality of service in the wire or wireless IP network when Mobile Nodes (MNs) move between IPv6 networks. The handover procedure includes discovery of the new prefix on the foreign subnet, establishment of the new Care-of Address (CoA), and notification of the new CoA of the MN to the Home Agent (HA) and Correspondent Nodes (CNs). The time during these phases is called handover latency. The MN cannot receive any packet during the handover procedure because neither the HA nor the CNs know the current location of the $\mathrm{MN}$, and actually the $\mathrm{MN}$ is unreachable.
The HA transmits these packets to the MN once it receives the Binding Update message from the $\mathrm{MN}$ and creates Binding Cache for the MN. As a result, applications on the $\mathrm{MN}$ are interrupted during the handover procedure. Therefore, reducing the handover latency is of significant importance for carrying uninterrupted real-time services like VoIP.

Handover latency of MIPv6 is the main cause resulting in performance degradation and service interruption. Some enhancements have been proposed to decrease handover latency such as fast handover (FMIPv6), hierarchical MIPv6 (HMIPv6) and fast handover for HMIPv6 (FHMIPv6). There are four phases included in MIPv6 handover procedure. They are Movement Detection, CoA Configuration, Home agent Registration and Route Optimization. It is essential to know the latency in each handover phase and the reason for the latency in order to determine the performance bottleneck and guide enhancement design for MIPv6.

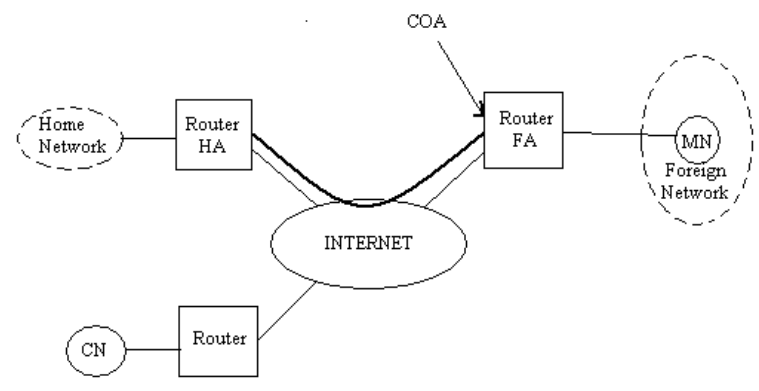

Fig 1.Architecture of mobile IP

\section{BACKGROUND}

A Handoff is the process that allows a Mobile Node (while that Mobile Node has a communication running) to change its point of attachment from an Access Router (AR) to another. Handoff is carried out as level 3 (Network layer) Handoff and level 2 (Connection layer) Handoff, according to the OSI model. The layer 2 Handoff is the operation carried out by a Mobile Node, which changes of radio access point. This Handoff can generate or not a Handoff of superior layer according to the wired connection of radio access points (if those are on the same network connection or not).

In the case of a Handoff of level 3, Mobile Node needs to acquire a new IP address. It requires registration messages, which can cause a communication interruption. The time of interruption can increase if the number of users increases. This will be awkward for the real time applications or delay 
sensitive traffic such as the voice over IP and video streaming.That's why a certain number of mobility protocols were presented to improve the performances of Mobile IP and in particular the Handoff process. These protocols are made for environments where the MIPv6 specifications such as signalization overload, packets loss, and data delivery delay are insufficient. These delays are directly related to the round-trip time of registration messages.

\section{A. IPv6 Background}

A Handoff is the process that allows a Mobile Node (while that Mobile Node has a communication running) to change its point of attachment from an Access Router (AR) to another. To solve the mobility problem of IP a standard was proposed, namely mobile IP. The components of the mobile IP protocol are:

MN: Mobile Node (sometimes called Mobile Host), this is the node that changes location.

$\mathrm{HN}$ : Home Network, the network in which the Mobile Node's (MN) home agent is positioned. This is the network in which the permanent address of the $\mathrm{MN}$ is located.

HA: Home Agent, which is in the router of the Home Network (HN).

FN: Foreign Network, the network that the $\mathrm{MN}$ is currently in.

FA: Foreign Agent, which is in the router of the Foreign Network (FN).

$\mathrm{CN}$ : Corresponding Node (sometime called Corresponding Host), the node that the $\mathrm{MN}$ is communicating with.

CoA: Care-of-Address, the temporary IP address that the Home Agent (HA) can use to contact the MN, while it resides in the FN. This CoA usually points to the Foreign Agent (FA), although it might sometimes point directly to the MN.

When a host moves and attaches itself to another network, it needs to obtain a new IP address. With this change of IP address, all existing connections to the mobile host terminates, as the IP routing mechanisms cannot deliver the data to the correct end-point. Mobile IPv4 overcomes this by introducing a level of indirection at the network (IP) layer. It deploys a home agent that intercepts packets from the correspondent host and redirects these packets by tunneling them to the mobile node via a foreign agent in the visiting network [1]. This approach ensures correspondent host transparency and only requires the mobile node to update its location to the home agent when changing in between networks. However, initiating this indirection requires a timely home network registration process and an address resolution procedure. These have been shown to result in long handover latencies, which leads to packet loss and sever TCP performance degradation. Two ways of overcoming these problems are to use a hierarchical architecture and to apply a technique known as fast-handoff.

\section{B. Literature survey}

Hierarchical schemes reduce handoff latency by employing a hierarchical network structure in minimizing the location update signaling with external networks. The hierarchical structure separates mobility into micro mobility (within one domain) and macro mobility (between domains). To support this, a special network agent entity, Mobility Anchor Point (MAP), is placed into the edges of the network [2]. It separates micro from macro mobility, by receiving packets on behalf of the mobile node that it is serving. Thus, when a host moves within a single domain, only location update to the MAP is necessary. This minimizes latency due to handoff between access routers by enabling the mobile node to perform only one local binding update, i.e., MAP binding update (BU), when changing its layer-3 (L3) IP address within the micro mobility domain as opposed to performing binding updates to its home agent and/or the corresponding nodes, which are potentially located in a distant external network.

Fast-handoff scheme reduces the handoff latency by allowing the mobile node to pre-configure a new care-of address (CoA) before it moves to a new access network. These concepts of the hierarchical framework and fast-handoff mechanism are incorporated into the IPv6 network.

\section{PROPOSED ARCHITECTURE}

\section{A. System Design}

Since the handover latency is reduced by both the schemes, in order to be more efficient the combination of both are performed. The main goal is to reduce the latency which is done by the following algorithm:

Step 1.The MN knows its movement toward NAR by the layer2 prehandover trigger (link layer address of NAR).

Step 2. Mobile node sends nCoA_REQ_MN with the information of MN \& PAR to MAP1.

Step 3.Micro Mobility:

3.1. MAP1 sends nCoA_REP message by generating nCoA and performing DAD to $\mathrm{MN}$.

3.2. $\mathrm{MN}$ request F-BU to MAP1.MAP1 starts handover procedure by sending HI to NAR. NAR sends the HACK to MAP1 and MAP1 sends FBACK to NAR and PAR.

Step 4.Macro Mobility:

4.1. After MAP receiving this message, it sends out the nCoA-REQ-MAP1 including the information of PAR and MN to MAP2.

4.2. Then MAP2 will find the right CoA for this $M N$ from the $\mathrm{CoA}$ table and send back a nCoA-REP including the new CoA to the MAP1.

4.3. Once receiving $\mathrm{nCoA}-\mathrm{REP}$ from MAP1, the MAP1 will advertise this $\mathrm{nCoA}$ to the $\mathrm{MN}$ by "nCoA-Adv" message.

4.4. At the same time, the "Binding Update to HA/CN (BU-HA/CN)" messages are sent to $\mathrm{HA}$ and $\mathrm{CN}$ separately by MAP1.

4.5. Also during binding update procedure, the "Binding Update Acknowledgement (BU-ACK)" should be replied by $\mathrm{HA} / \mathrm{CN}$.

Step 5. After the MN moves to the new domain of NAR, it will send a router solicitation with the FNA option followed by normal packet delivery. 


\section{B. The Fast Handover Scheme}

1) Scheme Overview: To reduce the handover latency, we merge the movement detection procedure and tunnel establishment procedure, and let PAR send $B U-H A / C N$ (Binding Update to $\mathrm{HA}$ and $\mathrm{CN}$ ) directly after PAR knows the new CoA. Also the DAD will not be performed during the period when the tunnel is established as in the predictive fast handover scheme, because DAD has already been performed beforehand. Thus the latency for performing DAD can be reduced. In the existing fast handover scheme, the binding update to the $\mathrm{HA} / \mathrm{CN}$ is performed after attaching to the NAR. However, in the proposed enhanced scheme, the binding update to

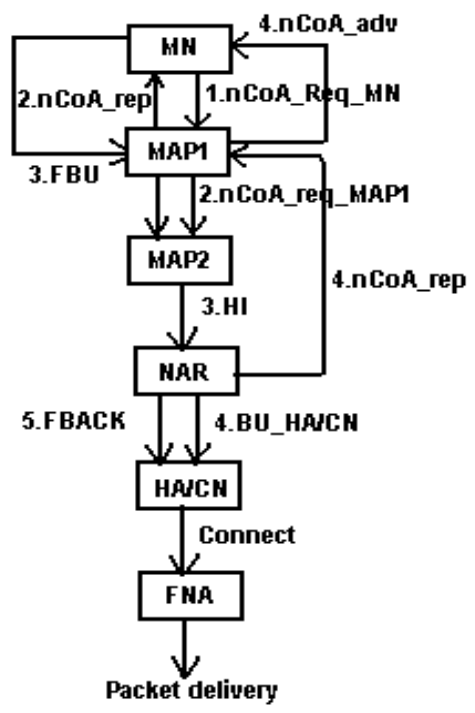

Fig 2 Packet flow in system architecture

$\mathrm{HA} / \mathrm{CN}$ is brought forward to the time before the layer 2 handover. Thus in our scheme, the completion time of BU of $\mathrm{HA} / \mathrm{CN}$ is advanced and the tunnel between PAR and NAR still exist shorter than in the fast handover scheme. Therefore, any packets passing to the MN through the tunnel can be sent to the MN directly without using the tunnel. That means he latency for these packets can be reduced. ote that in our scheme, binding update is performed by PAR not NAR, because it should be guaranteed that the nCoA has been known by PAR. That is, [2] if PAR has not received any information on $\mathrm{nCoA}$ but binding update has been performed, it will lead to topology error because the moving MN has two CoAs at the same time. On the other hand, after PAR knows the $\mathrm{nCoA}$, it will know that the MN has moved to the domain of NAR and make the current CoA unavailable to communicate with the MN directly [7]. At the same time, the nCoA is available to the corresponding nodes and HA by binding update. Therefore, there will be no problem on routability test after the $\mathrm{HA} / \mathrm{CN}$ receives $B U-H A / C N$ for the reason that there is only one $\mathrm{CoA}$ is in use for the $\mathrm{MN}$ at one time. Also we notice that even DAD procedure has not been brought forward as proposed, the binding update procedure can still be performed by PAR at the time point when the nCoA is known by PAR. What we need to do is to label this nCoA as a new one for an MN that will move from PAR to NAR. Thus even if the DAD fails at the beginning, the NAR still can identify the packets which are directed to the specified MN because the NAR can differentiate the packet destination by the information of nCoA, PAR and MN. By reducing the time taken for $\mathrm{DAD}$ operation the registration delay can be reduced very much.

2) Scheme Operations: In the following, the operations for the proposed enhanced fast handover scheme will be given.

Step 1: Firstly the MN will send the "new CoA Request from MN (nCoA-REQ-MN)" to PAR. After PAR receiving this message, it sends out the "New CoA Request from PAR (nCoA-REQ-PAR)" including the information of PAR and MN to NAR. Then NAR will find the right CoA for this MN from the CoA table and send back a "new CoA Reply (nCoA-REP)" including the new CoA to the PAR. Once receiving nCoA-REP from NAR, the PAR will advertise this $\mathrm{nCoA}$ to the MN by "nCoA-Adv" message. At the same time, the "Binding Update to HA/CN (BU-HA/CN)" messages are sent to HA and $\mathrm{CN}$ separately by PAR. Also during binding update procedure, the "Binding Update

Acknowledgement (BU-ACK)" should be replied by $\mathrm{HA} / \mathrm{CN}$.

Step 2: After the MN moves to the new domain of NAR, it will send a router solicitation with the FNA option. Then NAR will reply with a router advertisement with the FNA-ACK option.

\section{Hierarchical Setup}

One improvement to the original mobile IP protocol is a change in the architecture. Compared to the original architecture the new architecture is set up in a hierarchical fashion. As can be seen when compared to original architecture of mobile IP some new objects are introduced. The most important new object is the Domain Foreign Agent (DFA) which is connected to the router of the foreign domain (FD). In mobile IP each sub network has an FA connected to its router. This is not the case in hierarchical mobile IP, where the routers (R1 and R2) that are connected to the sub networks (FSN1 and FSN2) no longer have FAs. The FAs have been replaced by the DFA. Another change that has been made is the location of the CoA, which is now at the DFA. Whenever an MN moves from one sub network (FSN1) to another (FSN2) in the same domain the CoA, [3] that the HA uses to forward messages, does not change. Because the CoA does not change the $\mathrm{MN}$ does not have to send a binding update to the HA, which reduces the overall latency of the handoff. 


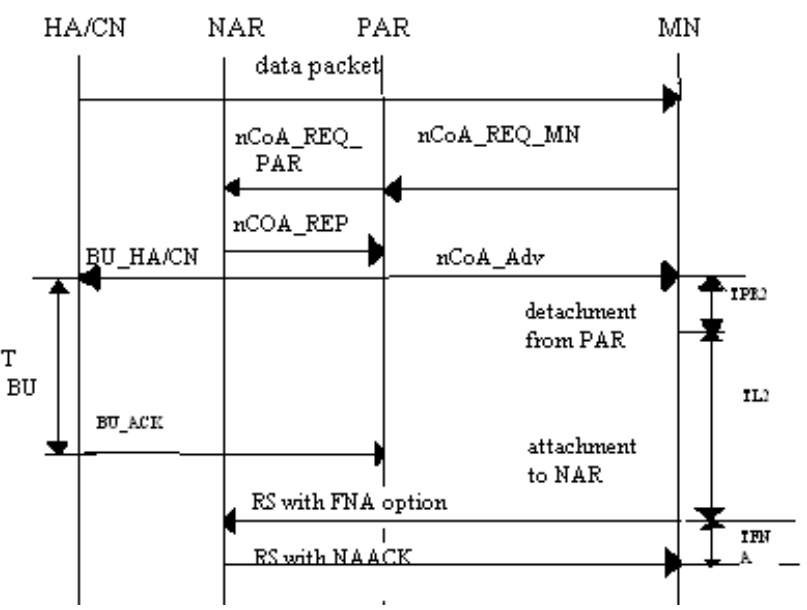

Fig 3 Timing graph for the fast handover scheme

The type of movement by the MN discussed above is called an intra-domain handoff. In this type of handoff the previous sub network is a member of the same domain as the next sub network. When the previous sub network is not part of the same domain as the next sub network an inter-domain handoff will take place. In an inter-domain handoff the MN will have to register its new CoA (as received from its new DFA) with its HA.

This occurs in the same way as it would in the regular IP protocol. Besides a reduction of the binding update latency the hierarchical set up also reduces the amount of traffic sent over the internet. Of course both these effects only occur during intra-domain handoff (movement within a domain), because inter-domain similarly to handoffs in traditional mobile IP handoffs

Hierarchical schemes separate mobility management into micro mobility and macro mobility or otherwise known as intra-domain mobility and inter-domain mobility respectively [6]. The central element of this framework is the inclusion of a special conceptual entity called Mobility Anchor Point (MAP)[4]. It is a router or a set of routers that maintains a binding between itself and mobile nodes currently visiting its domain. It is normally placed at the edges of a network, above the access routers, to receive packets on behalf of the mobile nodes attached to that network.

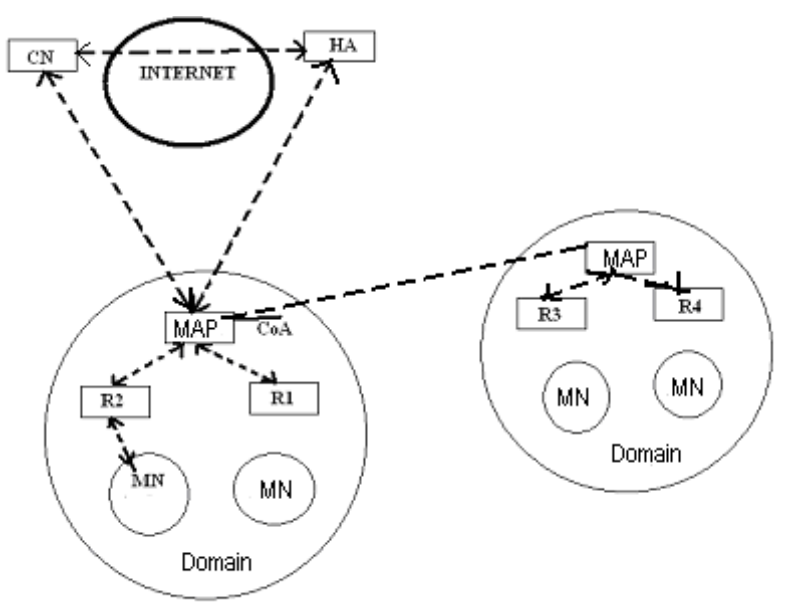

Fig 4 Hierarchical setup for Mobile IP
When a mobile node attaches itself to a new network, it registers with the MAP serving that network domain (MAP domain). The MAP acts as the local home agent for the mobile node. It intercepts all the packets addressed to the mobile node it serves and tunnels them to the corresponding on-link1 CoA of the mobile node. If the mobile node changes its current address within a local MAP domain, it only needs to register the new on-link address with the MAP since that the global CoA does not change [4]. If a mobile node moves into a new MAP domain, it needs to acquire a regional address ( $\mathrm{RCoA}$ ) and an on-link address (LCoA).

The mobile node then uses the new MAP's address as the RCoA, while the LCoA address can be formed.After forming these addresses, the mobile node sends a regular MIPv6 BU to the MAP, which will bind the mobile node's RCoA to its LCoA. If successful, the MAP will return a binding acknowledgement (BAck) to the mobile node indicating a successful registration [4].

In addition to the binding at the MAP, the mobile node must also register its new RCoA with its home agent by sending another BU that specifies the binding between its home address and the RCoA. Finally, it may send similar BU to its current corresponding nodes, specifying the binding between its home address and the RCoA.

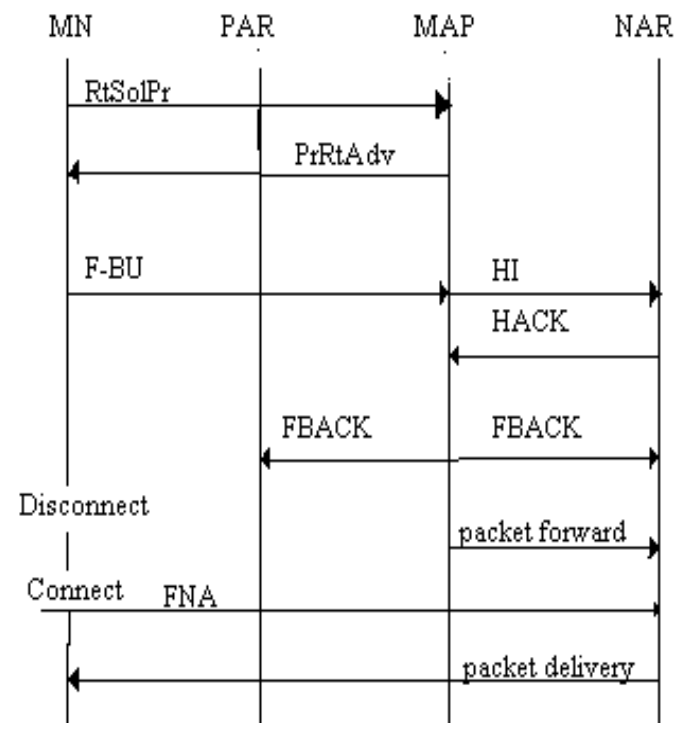

Fig 5 Timing graph for Hierarchical Mobile IPv6

\section{IMPLEMENTATION DETAILS}

The Network Simulator, ns, is used for the evaluation of the HMIPv6 with Fast-handoff mechanism. The standard ns distribution version ns-allinone $2.1 \mathrm{~b} 7 \mathrm{a}$ is patched with the freely available ns wireless extension module. This is further extended with our implementation of the MAP entity, the AR entity, the Mobile IPv6 binding mechanism, as well as protocols for the hierarchical management and the fast handoff mechanism [10].

Each simulation using Ns-2 has been performed which shows the test network topology used for simulation. The network consists of two MAPs and several access routers (ARs).The HA is connected to MAP1 via a link of 5Mbps 
bandwidth and 300ms transmission delay. The $\mathrm{CN}$ is connected to MAP2via a link of $5 \mathrm{Mbps}$ and 300ms delay. On the other hand, MAP1 is connected to MAP2 by the $5 \mathrm{Mbps}$ and $100 \mathrm{~ms}$ link, and each AR is connected to the MAPs by a $5 \mathrm{Mbps}$ and $40 \mathrm{~ms}$ link. The cell size of each node is assumed as $250 \mathrm{~m}$, and each cell has a $50 \mathrm{~m}$ overlap region. It is assumed that the layer 2 trigger for FMIPV6 operation occurs at $295.5 \mathrm{~m}$ and the link down at $250 \mathrm{~m}$. The link up timing is assumed at $20 \mathrm{~ms}$ after link down. In the simulation the MN starts to move from the location of $(100,100)$ at time10s, and then moves toward node 9 at a speed of $50 \mathrm{~m} / \mathrm{sec}$. Our simulation focused on evaluating the handover performance of the $\mathrm{MN}$ in the area spanning nodes 5 to 9 .

\section{Algorithm 1: Fast Handover}

CASE: MN sends the "new CoA Request from MN (nCoA-REQ-MN) to PAR.

IF: PAR receives this message

\section{THEN:}

a) It sends out the "New CoA Request from PAR (nCoA-REQ-PAR)" including the information of PAR and MN to NAR.

b) NAR will find the right CoA for this MN from the CoA table and send back a "new CoA Reply (nCoA-REP)" including the new CoA to the PAR.

c) Once receiving nCoA-REP from NAR, the PAR will advertise this $\mathrm{nCoA}$ to the $\mathrm{MN}$ by "nCoA-Adv" message.

d) At the same time, the "Binding Update to HA/CN (BU-HA/CN)" messages are sent to HA and $\mathrm{CN}$ separately by PAR.

e) Also during binding update procedure, the "Binding Update Acknowledgement (BU-ACK)" should be replied by $\mathrm{HA} / \mathrm{CN}$.

f) After the MN moves to the new domain of NAR, it will send a router solicitation with the FNA option.

g) Then NAR will reply with a router advertisement.

\section{Algorithm 2: Hierarchical setup}

CASE: The MN knows its movement toward NAR by the layer2 pre handover trigger (link layer address of NAR).

THEN:

a) MN sends RtSolPr to NAR.

b) MAP replies with PrRtAdv that contains the network prefix of NAR.

c) $\mathrm{MN}$ requests $\mathrm{FBU}$ to MAP with new $\mathrm{LCoA}$.

d) MAP sends HI message which includes request for verification of new LCoA and for establishment of bidirectional tunnel between MAP and NAR.

e) NAR performs DAD (Duplicate Address Detection) and responds with HACK to MAP.

f) MAP sends the result to MN by FBA.

IF: The MN gets connection to NAR

THEN: MN sends FNA message inorder to inform its presence.

\section{Algorithm 3: Fast Handover in Hierarchical Setup}

[1] The data packet is sent where the RtSolpr message is sent by the MN to MAP.

[2] MAP sends the HI (handover initiate) message to NAR, in which DAD process is completed in the NAR region. Hence the delay is reduced at the new access router region.

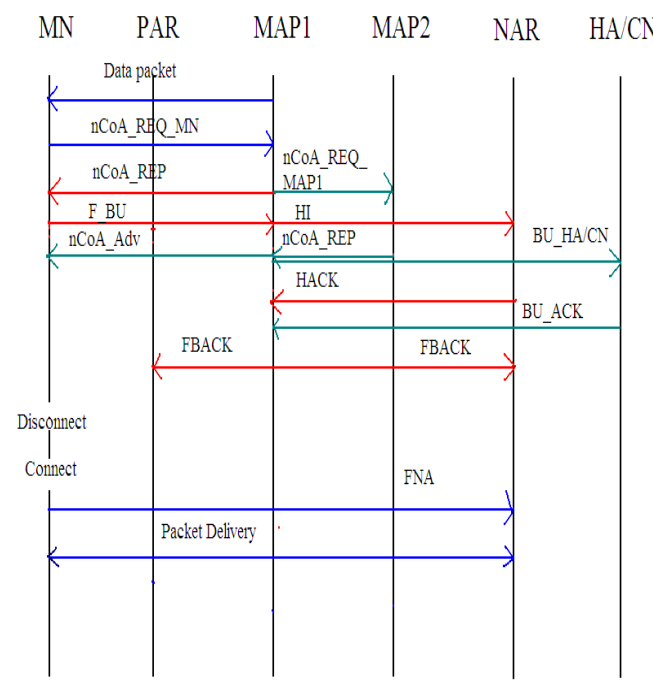

Fig 6 Timing graph for the fast handover scheme in hierarchical setup

[3] HACK message is sent by the NAR to the mobile node for the acknowledgment of the completion of the DAD process.

[4] The data packet is forwarded to the new access router which then sends the acknowledgment to the mobile node. Then the connection is established by the mobile node to the NAR and the packet is delivered

\section{PERFORMANCE ANALYSIS}

Once all the environment parameters are set and all the simulations are run it is time to evaluate the different scenarios that are presented in this work. The performance metrics that are considered are:

\section{A. Handoff Latency}

This is probably the most logical criterion, as this is the objective of most of the new or updated protocol suggestions. Decreasing the handoff latency generally improves the smoothness of the experience for the user. Of course there are other weighing factors when determining this smoothness, such as the amount of packet loss. For instance, a protocol with a 50 percent lower latency but 100 percent packet loss during the handoff will not be a good solution in most situations.

The network consists of two MAPs (MAP1 and MAP2) and several Access routers (AR).The access routers are connected to MAP's via a link of 5Mbps bandwidth and $40 \mathrm{~ms}$ transmission delay. The MAPs are connected via a link of $5 \mathrm{Mbps}$ bandwidth and $100 \mathrm{~ms}$ transmission delay. In the simulation the Mobile node (MN) starts to move from the location of $(340,370)$ at time 20 s and starts to move towards R2.At time 40s the Mobile node moves towards R3 and then to $\mathrm{R} 4$ in MAP2.The $\mathrm{CN}$ generates UDP protocol continuously from time $1 \mathrm{~s}$. The UDP traffic assumes 
Constant Bit Rate traffic (CBR) with a $0.01 \mathrm{~s}$ time interval. The packet size is 50 bytes.

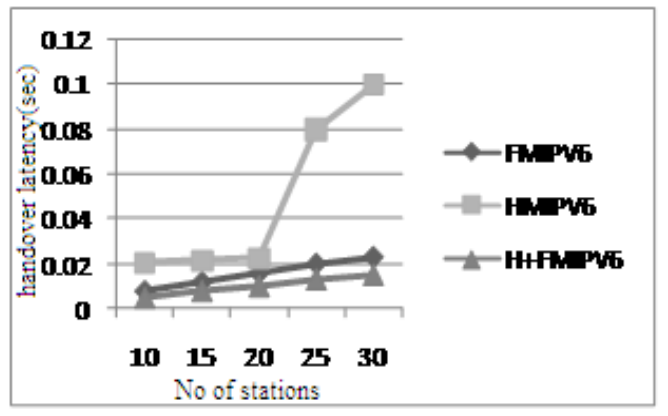

Fig 7 Latency Vs no of stations

TABLE 8 HANDOVER LATENCY OF VARIOUS MOBILE IPV6 VARIATIONS

\begin{tabular}{|c|c|}
\hline MIPv6 variations & Handover Latency(ms) \\
\hline HMIPv6 & 326 \\
\hline FMIPv6 & 358 \\
\hline F+HMIPv6 & 270 \\
\hline
\end{tabular}

When Layer 2 handover is performed the Mobile Node must proceed with new care-off address establishment. It must send binding updates to the Home Agent and the Corresponding Nodes that is needed. It also may need to send a Fast Binding Update to its old Access Router, unless it has a positive indication that the old Access Router already has made the appropriate binding cache modifications. These details may include receipt of a Router Advertisement from the new Access Router. This is considered as one of the main reason for saving time over latency and the major advantage of taking up this architecture.

With the help of $\mathrm{x}$-graph we are able confirm the handover latency value which is relatively lesser in our implemented proposed architecture.

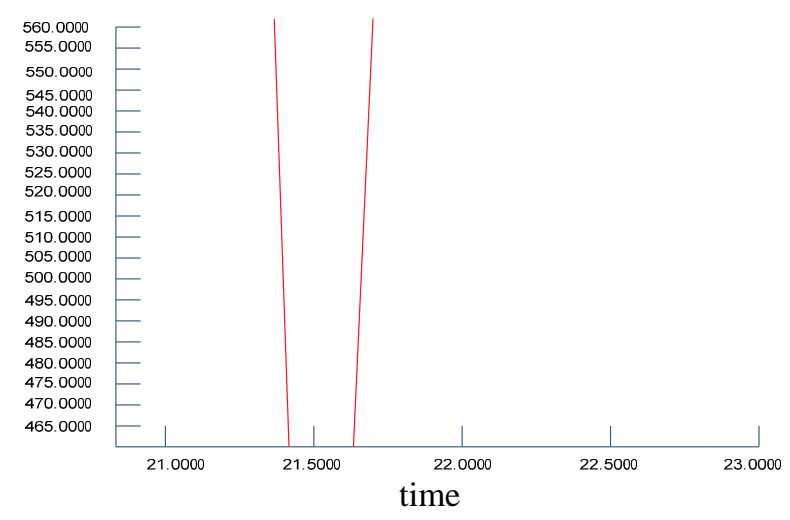

Fig 9 Packets received by MN

\section{CONCLUSION}

This project has described a new scheme for supporting a fast handover effectively. For analysis,F-HMIPV6 is compared to the simple combination of FMIPV6 and HMIPV6 in terms of handover latency. In this scheme, fast handoff mechanism is implemented in hierarchical set up, within a domain hierarchical setup can be used to reduce the handover latency and between networks fast handover scheme can be used to reduce the handover latency. Simulation of fast handover mobile IPv6 is been done.By reducing the handoff delay the packet loss also can be reduced and hence the Qos of VOIP can be improved. The effective operation between MAP is left for further study.

\section{REFERENCES}

[1] Ruidong Li, Jie Li, Kui Wu, and Jiang Xie, ”An Enhanced Fast Handover with Low Latency for Mobile IPV6”, IEEE, Januarary 2008.

[2] I. F. Akyildiz, J. Xie, and S. Mohanty, "A survey of mobility management in next-generation all-IP-based wireless systems," IEEE Wireless Commun., Aug. 2006.

[3] Hee Young Jung, Eun Ah Kim, JongWha Yi, and Hyeong Ho Lee "A Scheme for Supporting Fast Handover in Heirarchial Mobile IPv6 Networks", ETRI J., vol.27, no.6, Dec.2005, pp.798-801.

[4] Castelluccia, C. "HMIPv6: A hierarchical mobile IPv6 proposal", ACM SIGMOBILE Mobile Computing and Communications Review, volume 4, pp.48-59, 2005.

[5] W. Wang, S. C. Liew, and V. O. K. Li, "Solutions to performance problems in VoIP over a 802.11 wireless LAN," IEEE Trans. Veh.Technol., pp. 366-384, 2005.

[6] H.Soliman, C.Castelluccia, K.ElMalki, and L. Bellier, "Hierarchical mobile IPv6 mobility management (HMIPv6)," RFC 4140, Aug. 2005.

[7] Rajeev Koodli, Fast Handovers for Mobile IPv6, IETF RFC 4068, July 2005.

[8] Chen,H.,Zhang,J., "Prep-Binding of Fast Handovers for Mobile IPv6", Internet Draft, MIPSHOP Working Group, 2006.

[9] Kodaly R.,Perkins, C. "Mobile IPv6 Fast Handovers", Internet Draft, Mobile IPv6 Working Group, 2006.

[10] J.Widmer(2008) Extensions to the ns Network Simulator webpage on ICSI.BERKELY.[Online]

[11] Available.http://www.icsi.berkeley.edu/widmer/mnav/ns-estension/.

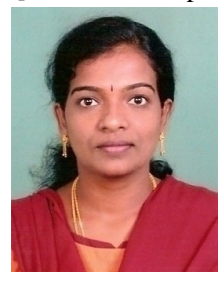
V.Berlin Hency Received her BE degree in Electronics and communication Engineering from Manonmaniam Sundaranar University India, ME degree in Applied Electronics from Sathyabama University India, Pursuing her $\mathrm{PhD}$ Degree in Information and Communication Engineering from Anna University,India. She is working as a lecturer in MIT campus, Anna University, Chennai, India. Her research interests include wireless multimedia networks, mobility management, mobile computing, and mobile IP.She is a member of IACSIT and the life member of Indian Society for Technical Education(ISTE)

Christina J, Dhushanthini A, Aiswariya V.T are the final year BE (Information Technology) students of MIT campus, Anna University,Chennai,India

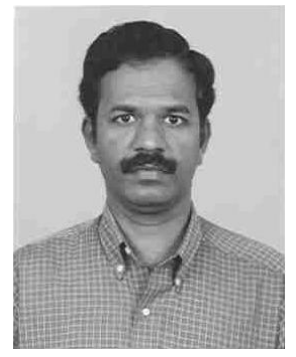

Dr.D.Sridharan received his B.Tech. degree in Electronics Engineering and M.E.degree in Electronics Engineering from Madras Institute of Technology, Anna University in the years 1991 and 1993 respectively $\mathrm{He}$ got his $\mathrm{Ph} . \mathrm{D}$ degree in the Faculty of Information and Communication Engineering, Anna University in 2005. He is currently working as Assistant Professor in the Department of Electronics and Communication Engineering, CEG Campus, Anna University, Chennai, India. He was awarded the Young Scientist Research Fellowship by SERC of Department of Science and Technology, Government of India. His present research interests include Internet Technology, Network Security, Distributed Computing and VLSI for wireless Communications. He has published more than 25 papers in National/International Conferences and Journals. He has visited USA, Italy, Germany, Singapore, Hong Kong and Dubai to participate and present his research papers and he has also attended a number of workshops sponsored by UNESCO. He is a life member of Institution of Electronics and Telecommunication Engineers (IETE), Indian Society for Technical Education(ISTE)and Computer Society of India(CSI). Presently he is working on a research project on Wireless Sensor Network sponsored by Department of Atomic Energy 